\title{
The rotational evolution of young low mass stars
}

\author{
Jérôme Bouvier \\ Laboratoire d'Astrophysique, Observatoire de Grenoble, Université Joseph Fourier, \\ B.P.53, 38041 Grenoble, Cedex 9, France \\ email: jbouvier@obs.ujf-grenoble.fr
}

\begin{abstract}
Star-disk interaction is thought to drive the angular momentum evolution of young stars. In this review, I present the latest results obtained on the rotational properties of low mass and very low mass pre-main sequence stars. I discuss the evidence for extremely efficient angular momentum removal over the first few Myr of pre-main sequence evolution and describe recent results that support an accretion-driven braking mechanism. Angular momentum evolution models are presented and their implication for accretion disk lifetimes discussed.
\end{abstract}

Keywords. Stars: pre-main-sequence, stars: rotation, accretion, accretion disks.

\section{Introduction}

Star-disk interaction in young systems involves mass and angular momentum transfer. Mass transfer manifests itself by accretion onto the star and wind/jet outflows while angular momentum transfer is thought to drive the rotational evolution of young stars and is also seen in jet rotation (cf. T. Ray's review in this volume). The purpose of this review is to address the following issues : i) what do observations tell us about the rotational evolution of young stars, ii) what is the impact of star-disk interaction on angular momentum evolution during the pre-main sequence, and iii) how successful are models in accounting for the observed rotational evolution of young stars?

Section 2 describes the rotational properties of low mass $\left(0.2-1.0 \mathrm{M}_{\odot}\right)$ pre-main sequence stars. Recent results include the determination of rotation periods for hundreds of stars in young clusters spanning an age range from $\leqslant 1$ Myr to the zero-age main sequence and beyond. For the first time, these results provide a relatively well sampled age sequence, from which the rotational evolution of young stars can be read.

Since the early 90 's, it is commonly thought that the star-disk interaction is somehow reponsible for the low rotation rate of young stars. Section 3 discusses the issue of accretion-related angular momentum loss, a process which should manifest itself by accreting stars being, on average, slower rotators than non accreting ones. Conflicting evidence for such a connection has been reported and is revisited here in the light of new results.

Section 4 provides a brief status of current angular momentum evolution models. Most models rely on the same simplified assumptions and still await to be upgraded to an actual physical description of the angular momentum loss processes acting during the pre-main sequence. The implications of these models for the lifetime of accretion disks are discussed. 
(a) NGC 2264

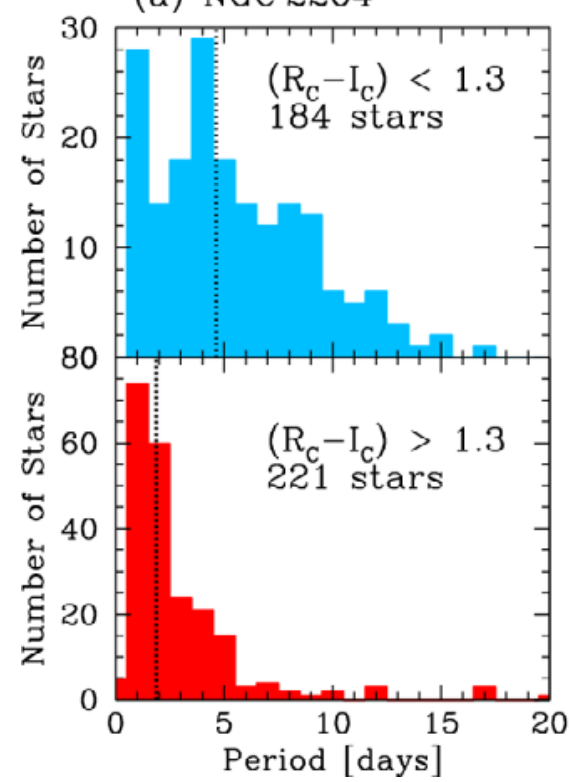

(b) ONC

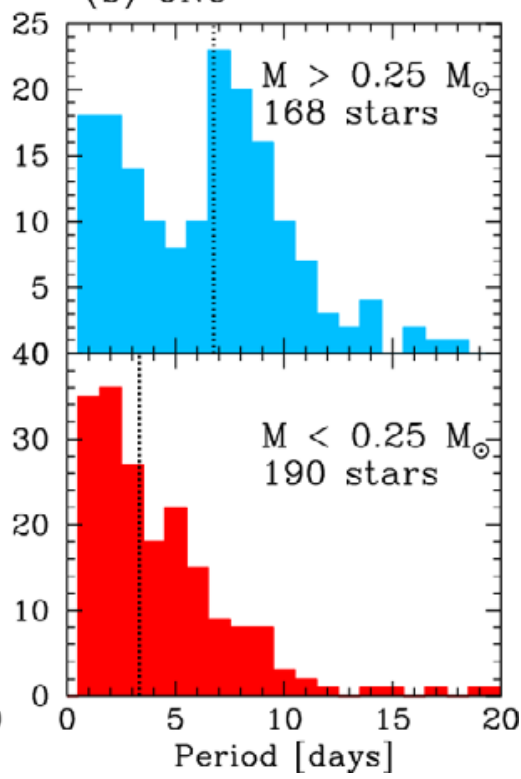

Figure 1. The rotational period distributions of low mass (top) and very low mass (bottom) stars in the $\sim 2-4$ Myr NGC 2264 cluster (left) and in the $\sim 1$ Myr Orion Nebulae Cluster (right). From Lamm et al. (2005).

\section{The rotational periods of young low mass stars}

In the last 10 years, rotational periods have been measured for hundreds of low mass pre-main sequence stars in various star forming regions and young open clusters. Rotational periods are derived by detecting a periodic component in the star's photometric variability, which results from the modulation of the star's brightness by surface spots. While this observational approach is time consuming, requiring the photometric monitoring of hundreds of stars over a timescale of several weeks, it is also far superior to spectroscopic $v \sin i$ measurements as the photometrically-derived rotational period is not affected by inclination effects. Moreover, the star's angular velocity, a key physical parameter of star-disk interaction models, relates directly to the rotational period $(\Omega=2 \pi / P)$, without having to resort to the poorly known stellar radius. These properties have been prime motivations for lauching large scale photometric monitoring campaigns targetting star forming regions with the hope to derive the rotational period distribution of statistically significant samples of pre-main sequence stars at various stages of their evolution.

The first large scale photometric monitoring campaign by Choi \& Herbst (1996) revealed the peculiar bimodal distribution of periods for young stars in the Orion Nebulae Cluster (ONC). The distribution of periods at an age of about 1 Myr exhibits two peaks, one located at a period of about 8 days, the other at a period of about 2-3 days, with approximately twice as many slow rotators as fast ones. While this result was disputed a few years later (Stassun et al. 1999), the bimodal distribution was eventually confirmed, with peaks near 2 and 8 days, for ONC stars more massive than $0.25 \mathrm{M}_{\odot}$ (Herbst et al. 2002). Interestingly, the same study also showed that the bimodality does not extend to lower mass stars whose period distribution exhibits a single peak at fast rotation with a mean period of about 2 days. This was the first hint at possibly different 
rotational properties between low mass $\left(0.3-1.0 \mathrm{M}_{\odot}\right)$ and very low mass $\left(\leqslant 0.3 \mathrm{M}_{\odot}\right)$ young stars.

One of the first comparative studies between 2 clusters of different ages came from the measurement of hundreds of rotational periods for low mass stars in NGC 2264 (Lamm et al. 2005). With an estimated age of 2-4 Myr, NGC 2264 is slightly older than ONC, thus allowing a first attempt to trace the evolution of rotation at the start of pre-main sequence evolution. The distribution of periods for ONC and NGC 2264 are shown in Figure 1 for low mass and very low mass stars, with a dividing line around $0.25 \mathrm{M}_{\odot}$. In each mass bin, the rotational distributions for the 2 clusters have a similar shape, bimodal for low mass stars and single-peaked for very low mass stars. However, on a timescale of a few Myr from ONC to NGC 2264, the peaks of the distributions have shifted towards shorter periods, indicative of stellar spin up by a factor of about 1.5 to 2 . Within the uncertainties on the age of the 2 clusters and assuming that the initial period distributions were similar in both clusters, this amount of spin up is consistent with stellar contraction and angular momentum conservation (Lamm et al. 2005). While most stars appear to spin up between ONC and NGC 2264, a tail of slow rotators remains, which suggests efficient braking for a fraction of low mass stars during the first $2 \mathrm{Myr}$ of their evolution.

A major leap in the study of angular momentum evolution of young stars recently came from the derivation of hundreds of rotational periods for low mass and very low mass stars in various clusters spanning an age range from 5 to $200 \mathrm{Myr}$. The Monitor project (Aigrain et al. 2007; Irwin et al. 2007a) aims at detecting eclipsing binaries and planetary transits in young low mass stars. As a by-product of intense photometric monitoring campaigns, precise rotational periods are derived for the young populations of these clusters (Irwin et al. 2006, 2007b). The period distributions derived for stars in the mass range 0.1$1.0 \mathrm{M}_{\odot}$ are shown in Figure 2 over the age range 1-200 Myr. The shape of the period distribution evolves drastically over the first 40 Myr. Starting from ONC at 1 Myr, the very low mass stars $\left(\mathrm{M} \leqslant 0.25 \mathrm{M}_{\odot}\right)$ appear to continuously spin up, from a median initial period of 2-3 days at $1 \mathrm{Myr}$ to a median period of 0.5-0.7 days at $40 \mathrm{Myr}$ and even shorter at $150 \mathrm{Myr}$. The rapid convergence of very low mass stars towards fast rotation, with no slow rotators left, is quite remarkable indeed.

As a group, higher mass stars $\left(\mathrm{M} \geqslant 0.5 \mathrm{M}_{\odot}\right)$ also tend to spin up during PMS evolution, though not as much as very low mass stars, with a median initial period of about 6 days at $1 \mathrm{Myr}$ shortening to about 3 days at $40 \mathrm{Myr}$. However, the initial bimodal distribution of periods seen for stars in this mass range remains visible over the whole PMS evolution. A fraction of initially slowly rotating stars, with periods of 8-15 days at $1 \mathrm{Myr}$, retain similar periods up to $5 \mathrm{Myr}$ and experience only mild spin up later on, with periods in the range 5-10 days at $40 \mathrm{Myr}$. Meanwhile, the initially fast rotators, with periods of 1-3 days at $1 \mathrm{Myr}$, spin up continuously with the shortest periods decreasing from $0.9 \mathrm{~d}$ at $1 \mathrm{Myr}$, to $0.5 \mathrm{~d}$ at $5 \mathrm{Myr}$ and $0.2 \mathrm{~d}$ at $40 \mathrm{Myr}$.

Recent results thus converge in indicating a somewhat different rotational evolution between low mass and very low mass stars during the pre-main sequence. Most very low mass stars $\left(\mathrm{M} \leqslant 0.25 \mathrm{M}_{\odot}\right)$ start their evolution as fast rotators and spin up continuously as they descend their Hayashi tracks and approach the ZAMS. In contrast, a significant fraction of low mass stars $\left(0.3-1.0 \mathrm{M}_{\odot}\right)$ experience milder spin up during their pre-main sequence evolution. Indeed, some appear to evolve at constant angular velocity for the first $5 \mathrm{Myr}$ at least (see also Rebull et al. 2004). Clearly, a very efficient brake must be at work to extract angular momentum in these young stars in order to prevent them from spinning up in spite of stellar contraction. 


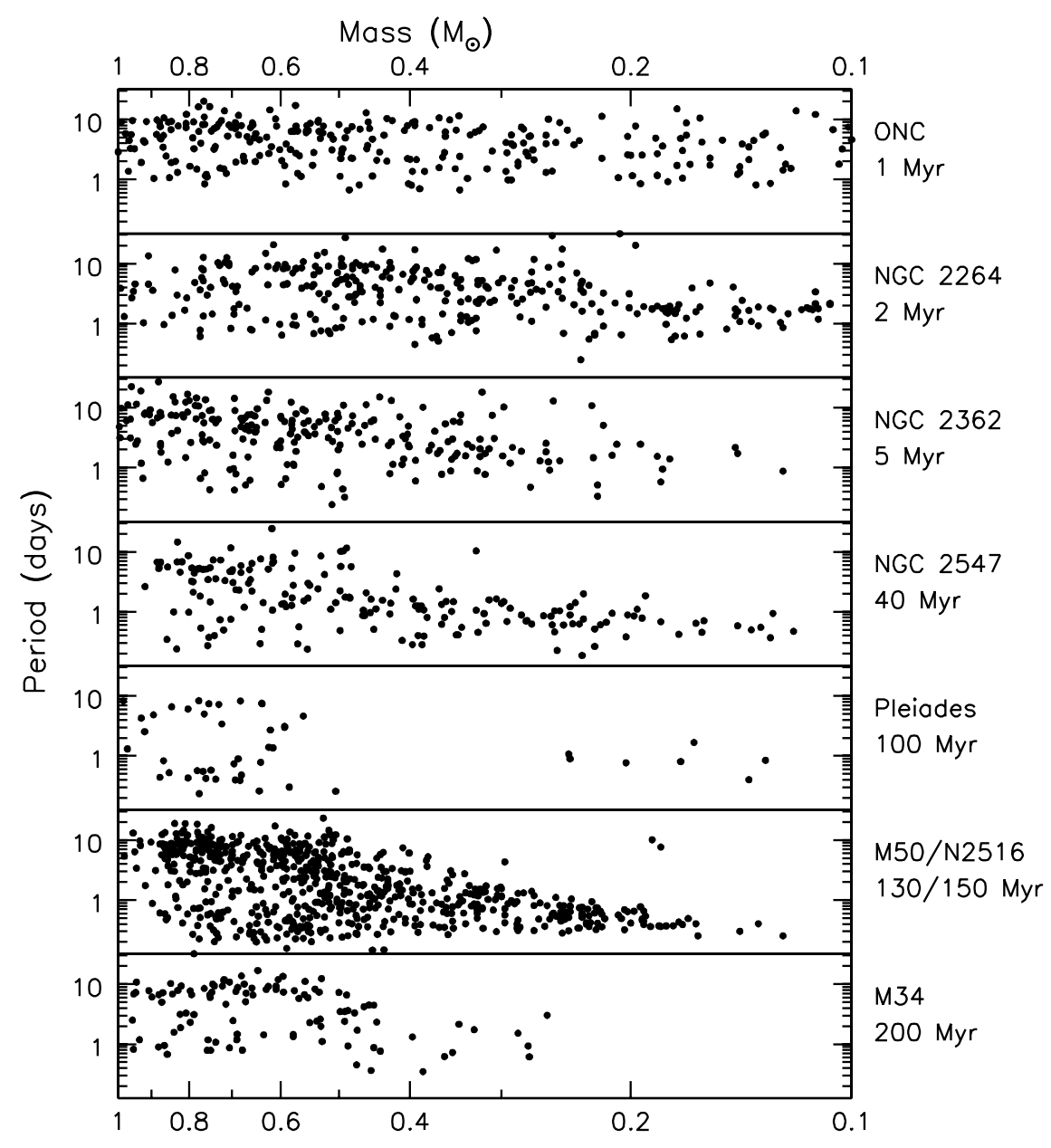

Figure 2. The rotational period distribution of low mass and very low mass stars in various clusters in the age range from 1 to 200 Myr. Note the evolution of the shape of the period distribution as a function of time, especially at very low masses. Adapted from Irwin et al. (2007b) and references therein.

\section{Is PMS braking related to the accretion process?}

On the main sequence, solar-type stars are braked at a low pace by their magnetized winds. Even though magnified versions of solar-type winds probably exist in the magnetically active low mass PMS stars, the associated braking timescale is much longer than the Kelvin-Helmotz contraction timescale (Bouvier et al. 1997). As a result, these winds cannot prevent the star from spinning up as it contracts towards the ZAMS (see Matt, this volume).

As an alternative, following models developped for X-ray binaries, Königl (1991) suggested that the magnetic star-disk interaction could regulate the angular momentum of the star. This idea has since been developped in a variety of MHD models where angular momentum is extracted from the star by the magnetic field and carried away by the disk or by an accretion-driven wind (see the contributions by Shu, Fendt, Romanova, and 


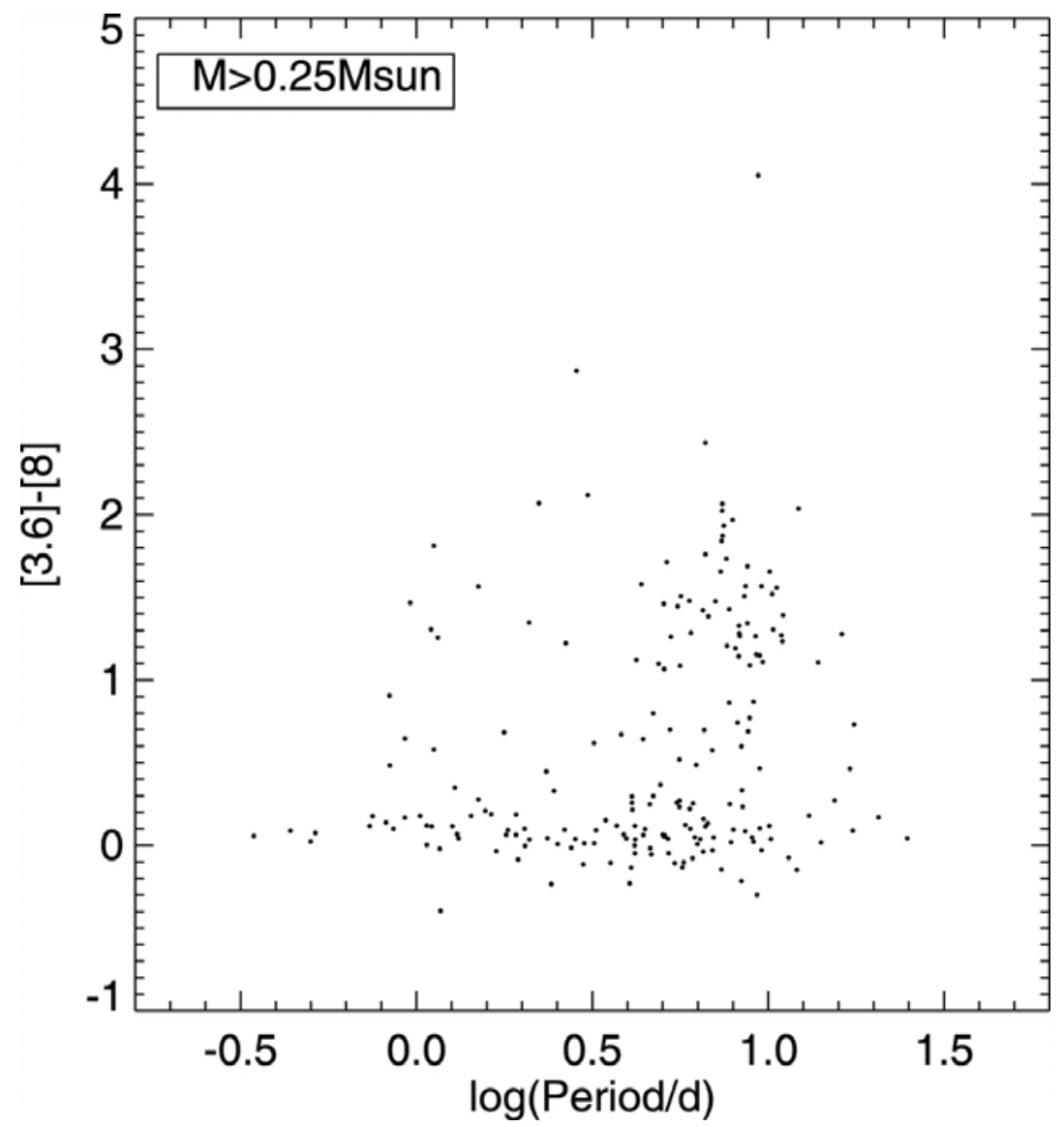

Figure 3. Spitzer [3.6]-[8.0] color excess as a function of rotational period for Orion low mass stars $\left(\mathrm{M} \geqslant 0.25 \mathrm{M}_{\odot}\right)$. Stars with disks have [3.6]-[8.0] $\geqslant 1$. From Rebull et al. (2006).

Ferreira in this volume). In these models, the star is thus braked as long as it accretes from its disk.

Irrespective of the specific underlying physical model (X-wind, disk locking, accretiondriven disk winds or stellar winds), any accretion-related angular momentum loss process should reveal itself as accreting stars being, on average, slower rotators than non accreting ones. Such a relationship between rotation and accretion has therefore been actively searched for. A first hint that such a correlation may exist was reported for a limited sample of T Tauri stars in Taurus with known rotational period and IR excess (Edwards et al., 1993). A larger scale study of ONC stars however revealed no such correlation (Stassun et al. 1999).

One difficulty in searching for rotation-accretion connection, besides statistical robustness, is to identify an unambiguous diagnostics of accretion onto the star. The often used near-IR and mid-IR excesses are somewhat ambiguous in this respect as they can arise from passive, i.e., non accreting, disks. The UV continuum excess is a more direct measurement of accretion onto the star but has been measured for too few young stars to be useful. $\mathrm{H}_{\alpha}$ line emission is a proxy of accretion onto the stellar surface, as long as it can be shown that the line flux or width exceeds the chromospheric emission component. In some regions with strong nebular $\mathrm{H}_{\alpha}$ emission, like ONC, the interpretation of the $\mathrm{H}_{\alpha}$ line is however not straighforward. 
Keeping in mind the limitations associated to the various accretion diagnostics, a number of recent studies have investigated the accretion-rotation connection for relatively large samples of young stars with known rotational periods. Lamm et al. (2005) used $\mathrm{H}_{\alpha}$ equivalent width to distinguish between accreting and non accreting $\mathrm{T}$ Tauri stars in NGC 2264 and found the former to be on average slower rotators than the latter. Similarly, Rebull et al. (2006) found that Orion low mass stars with longer rotational periods were more likely than those with short periods to exhibit a continuum mid-IR excess indicative of disks. At odds with these results, Cieza \& Baliber (2006) failed to find any correlation between accretion and rotation for IC 348 low mass stars, even though they used the same accretion diagnostics as Rebull et al. (2006).

These conflicting results on whether or not a relationship exists between rotation and accretion in young stars may have a number of causes. Statistical robustness is still an issue. Rebull et al. (2004) demonstrated from Monte Carlo simulations that samples of at least 400 stars per mass bin and a perfect knowledge of their accretion status would be needed to distinguish between the period distribution of accreting and non accreting stars. Current samples are still about 10 times smaller per mass bin and ambiguity remains for a fraction of stars regarding their accretion status.

Rebull et al.'s (2006) results for Orion low mass stars are shown in Figure 3. Four groups of stars can be distinguished in the mid-IR excess versus rotational period diagram, of which only 2 fulfill the expectations of accretion-regulated angular momentum evolution: slow rotators with strong IR excess, i.e., stars still actively accreting from their disk and thus prevented from spinning up, and fast rotators with no mid-IR excess, i.e., diskless stars free to spin up as they contract. The third group consists of only a few stars with short periods which exhibit a significant mid-IR excess. Owing to the small number of stars in this group, it is conceivable that they represent a transient state of fast rotation, on a timescale of $0.1 \mathrm{Myr}$, in spite of disk accretion. Interestingly, this group might hint at a discontinuous PMS braking process (e.g Popham 1996). The fourth group consists of a significant fraction of slow rotators with no mid-IR excess. Thus, among stars without IR excess, about half have periods longer than 5 days.

This latter group is the most puzzling as these stars lack evidence for a disk and yet rotate slowly. They are often interpreted as having dissipated their disk recently, and thus have not had time yet to spin up. However, similar groups of slowly rotating and apparently non accreting stars are observed in other star forming regions, over the age range from $\leqslant 1$ Myr (e.g. ONC) to $\sim 5 \mathrm{Myr}$ (e.g. Taurus). The spin up rate scales as $\mathrm{R}_{\text {star }}^{2}$ during the first few Myr, as long as the star remains fully convective, and increases later on as the radiative core develops. Thus, assuming an average initial period of 8 days at $1 \mathrm{Myr}$, a star would spin up to periods shorter than 5 days in about 1 Myr. One thus has to assume that, in each of the observed regions, a significant fraction of stars $(\sim 30 \%)$ were released from their disk less than a Myr ago over the age range 1-5 Myr.

Overall, signatures of the accretion-rotation connection, as expected from accretionregulated angular momentum evolution, have been recently reported. However, some intriguing results remain. In particular, a significant subgroup of apparently non accreting stars have long periods, which does not fit the accretion-regulated angular momentum scenario. Clearly, further characterization of these slow rotators is needed to assess their actual accretion status. Also, additional Monte Carlo simulations would help to clarify the interpretation of the accretion-rotation diagrams over PMS evolution timescales. 

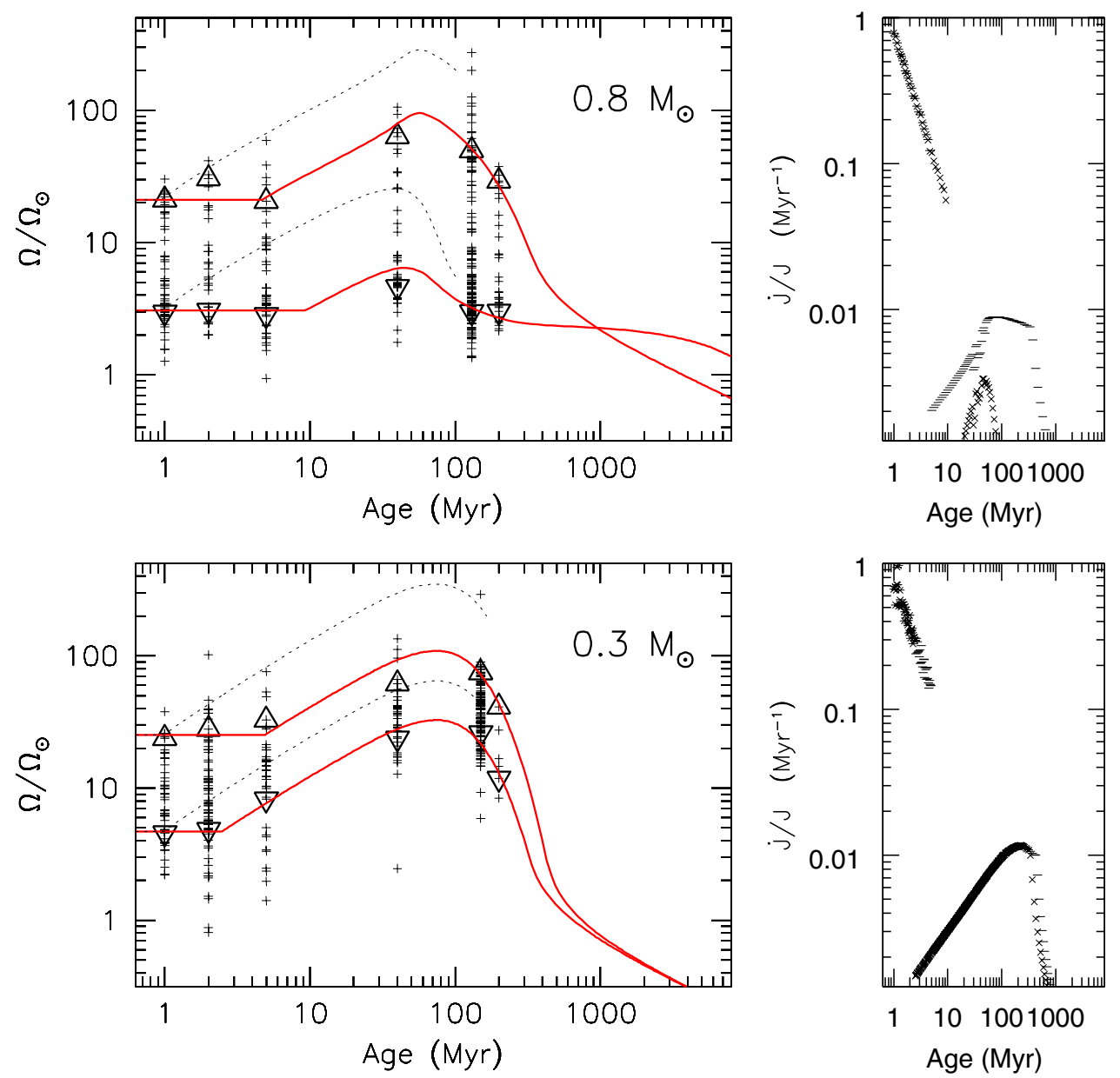

Figure 4. Angular momentum evolution models for $0.8 \mathrm{M}_{\odot}$ and $0.3 \mathrm{M}_{\odot}$ stars. Left : The distribution of rotational periods for stars in the mass range $0.7-0.9 \mathrm{M}_{\odot}($ top $)$ and $0.20-0.35 \mathrm{M}_{\odot}$ (bottom) are shown by crosses. Large triangles indicate the 25th and 90th percentiles of the distribution in each cluster. Models for slow and fast rotators are shown by solid lines. The initial rotational periods at $1 \mathrm{Myr}$ are 1.2 and 8.3 days for $0.8 \mathrm{M}_{\odot}$ stars $($ top $)$ and 1.0 and 5.4 days for $0.3 \mathrm{M}_{\odot}$ stars (bottom). Disk locking timescales range from 2.5 to $10 \mathrm{Myr}$. Once the star is eventually released from its disk, solar-type winds are the only source of angular momentum loss. Dotted lines show similar models over the first $200 \mathrm{Myr}$ for stars released from their disk at 1 Myr. See Bouvier, Forestini \& Allain (1997) and Allain (1998) for the details of the models. Right : Angular momentum loss rate (per Myr) for the models shown in the left panels. In order to evolve at constant angular velocity during the first 5-10 Myr in spite of contraction, the star must lose, on average, about a third of its angular momentum per Myr. The angular momentum loss rate from "disk locking" is over a hundred times more efficient than solar-type winds to brake the star during the early PMS.

\section{Models of angular momentum evolution and disk lifetimes}

Models of angular momentum evolution during the pre-main sequence (PMS) and on the zero-age main sequence (ZAMS) have been developped in the mid- and late-90's and have not much progressed since then. These models rely on 2 processes to remove angular momentum from the star : i) the so-called "disk-locking", which assumes extremely 
efficient angular momentum removal from the star as long as it magnetically interacts with its disk, thus forcing the star to evolve at constant angular velocity in spite of rapid contraction (Collier Cameron, Campbell \& Quaintrell 1995; Bouvier, Allain \& Forestini 1997), ii) a magnetized solar-type stellar wind which extracts angular momentum from the central star, acting simultaneously as disk locking although at a much weaker rate, and whose efficiency saturates at high velocity (Kawaler 1988; Keppens, McGregor \& Charbonneau 1995). Clearly, these models still lack a real physical description of the angular momentum removal processes and use instead semi-empirical parametrized braking laws. In spite of this limitation, this class of models have been reasonably successful in reproducing the global trends seen in the rotational evolution of young low mass stars (see, e.g., Bouvier, Allain \& Forestini 1997; Allain 1998; Sills et al. 2000).

Fig. 4 shows angular momentum evolution models computed for 0.3 and $0.8 \mathrm{M}_{\odot}$ stars and compared to the most recent rotational datasets discussed in the previous sections. The models were computed for 2 initial periods in each mass bin, corresponding to slow and fast rotators, respectively, as observed at $1 \mathrm{Myr}$ in the ONC. For both 0.3 and $0.8 \mathrm{M}_{\odot}$, a fraction of stars appear to evolve at constant angular velocity for a few Myr, and up to $10 \mathrm{Myr}$, before being released from their disk. For comparison, models in which the stars are released from their disk at $1 \mathrm{Myr}$ are shown and predict velocities much higher than observed on the ZAMS. Hence, disk locking appears to be active for at least a few Myr in order to prevent stars from spinning up as they contract. Once the stars are eventually released from their disk, they spin up as they approach the ZAMS until angular momentum losses from solar-type stellar winds become efficient enough to brake them over a timescale of a few $100 \mathrm{Myr}$. Note that while these models reproduce reasonably well the rotational evolution of low mass stars during the PMS, they predict too strong angular momentum loss from magnetized winds for very low mass stars on the MS (cf. Sills et al. 2000).

The right panels of Fig. 4 illustrate the angular momentum loss rate experienced by slow and fast rotators in the 2 mass bins. The most striking feature seen in these plots is that in order to prevent the star from spinning up as it contracts during the first few Myr, about a third of the stellar angular momentum must be removed per Myr. Thus, a slowly rotating $0.8 \mathrm{M}_{\odot}$ star reduces its angular momentum by a factor of 5 between 1 and $10 \mathrm{Myr}$. Clearly, an extremely efficient brake must apply to the star as long as it accretes from its disk, far more efficient indeed than angular momentum losses due to solar-type winds. MHD star-disk interaction models have to face this challenge : not only the stardisk interaction does not spin the star up, but it actively brakes it. In other words, it is not enough to balance positive accretion torques by negative magnetic torques to reach a zero net flux of angular momentum onto the star. Instead, it is mandatory that the star-disk interaction process actually removes angular momentum from the star at a high rate, i.e. that the net torque on the star be strongly negative, if the star is to evolve at constant angular velocity in spite of contraction.

Finally, the comparison of models with observations suggests that the rotational velocity of low mass PMS stars is regulated over a timescale of a few Myr, typically from 2 to 10 Myr. Since the strong brake is thought to result from active star-disk interaction, this requires that disk accretion lasts over at least this timescale. Current estimates of disk lifetimes have been obtained from a variety of diagnostics, including near- and mid-IR excess, and $\mathrm{H}_{\alpha}$ emission line width. While the former probe both active and passive disks, the latter provides more direct evidence for accretion onto the star. The disk fraction derived from near-IR excess amounts to about 40-60\% at $1 \mathrm{Myr}$, with no disk remaining past 5 Myr (Hillenbrand 2005). When diagnosed from the more sensitive mid-IR excess and/or $\mathrm{H}_{\alpha}$ width measurements, longer disk survival times are obtained, 
with about $40-60 \%$ of stars still surrounded by circumstellar disks at $2 \mathrm{Myr}$, a fraction which decreases to about 10-25\% at 10 Myr (e.g. Damjanov et al. 2007; Lyo \& Lawson 2005; Jayawardhana et al. 2006). Interestingly, a recent study of the 8 Myr $\eta$ Cha cluster suggests a mean disk lifetime of 9 Myr for single low mass stars while binary systems seem to dissipate their disks on a timescale of $5 \mathrm{Myr}$ (Bouwman et al. 2007). No primordial disks appear to survive past 30 Myr (Gorlova et al. 2007).

\section{Conclusions}

New datasets now provide hundreds of rotational periods measured for low mass and very low mass stars in young clusters over the age range from $1 \mathrm{Myr}$ to $0.2 \mathrm{Gyr}$. The distribution of rotational periods shows a clear evolution over the pre-main sequence contraction timescale. While most stars tend to spin up as they descend their Hayashi tracks, a fraction retain constant angular velocity for a few million years. This provides clear evidence for a strong brake acting on the stars on a timescale of 2-10 Myr. The new data also confirm that very low mass stars $\left(\mathrm{M} \leqslant 0.3 \mathrm{M}_{\odot}\right)$ tend to suffer lower angular momentum losses than low mass ones $\left(0.3-1.0 \mathrm{M}_{\odot}\right)$. The early evolution at nearly constant angular velocity implies angular momentum loss rates much larger that those achievable by solar-type magnetized stellar winds. New empirical evidence has been recently reported which confirms that the magnetic star-disk interaction is indeed responsible for the braking of low mass stars at the start of their PMS evolution. These new results challenge the ability of current MHD star-disk interaction models to extract as much angular momentum from the young star as needed to prevent it from spinning up in spite of accretion and contraction. On the observational side, a critical time step still to be sampled in order to better constrain accretion disk lifetimes from rotational evolution is the 5-40 Myr range as most disks appear to dissipate on these timescales.

\section{Acknowledgements}

I would like to thank Jonathan Irwin for providing some of the Monitor data prior to publication.

\section{References}

Aigrain, S., Hodgkin, S., Irwin, J., Hebb, L., Irwin, M., Favata, F., Moraux, E., \& Pont, F. 2007, MNRAS, 375, 29

Allain, S. 1998, A\& A, 333, 629

Bouvier, J., Forestini, M., \& Allain, S. 1997, A\&A, 326, 1023

Bouwman, J., Lawson, W. A., Dominik, C., Feigelson, E. D., Henning, T., Tielens, A. G. G. M., \& Waters, L. B. F. M. 2006, ApJ (Letters), 653, L57

Choi, P. I., \& Herbst, W. 1996, AJ, 111, 283

Cieza, L., \& Baliber, N. 2006, ApJ, 649, 862

Collier Cameron, A., Campbell, C. G., \& Quaintrell, H. 1995, A\& A, 298, 133

Damjanov, I., Jayawardhana, R., Scholz, A., Ahmic, M., Nguyen, D. C., Brandeker, A., \& van Kerkwijk, M. H. 2007, ArXiv e-prints, 708, arXiv:0708.0266

Edwards, S., et al. 1993, AJ, 106, 372

Gorlova, N., Balog, Z., Rieke, G. H., Muzerolle, J., Su, K. Y. L., Ivanov, V. D., \& Young, E. T. 2007, ArXiv e-prints, 707, arXiv:0707.2827

Herbst, W., Bailer-Jones, C. A. L., Mundt, R., Meisenheimer, K., \& Wackermann, R. 2002, $A \mathscr{S} A, 396,513$

Hillenbrand, L. A. 2005, ArXiv Astrophysics e-prints, arXiv:astro-ph/0511083

Irwin, J., Irwin, M., Aigrain, S., Hodgkin, S., Hebb, L., \& Moraux, E. 2007a, MNRAS, 375, 1449 
Irwin, J., Aigrain, S., Hodgkin, S., Irwin, M., Bouvier, J., Clarke, C., Hebb, L., \& Moraux, E. 2006, MNRAS, 370, 954

Irwin, J., Hodgkin, S., Aigrain, S., Hebb, L., Bouvier, J., Clarke, C., Moraux, E., \& Bramich, D. M. 2007b, MNRAS, 377, 741

Jayawardhana, R., Coffey, J., Scholz, A., Brandeker, A., \& van Kerkwijk, M. H. 2006, ApJ, 648, 1206

Kawaler, S. D. 1988, ApJ, 333, 236

Keppens, R., MacGregor, K. B., \& Charbonneau, P. 1995, A\&A, 294, 469

Königl, A. 1991, ApJ (Letters), 370, L39

Lamm, M. H., Mundt, R., Bailer-Jones, C. A. L., \& Herbst, W. 2005, A\&\&A, 430, 1005

Popham, R. 1996, ApJ, 467, 749

Rebull, L. M., Wolff, S. C., \& Strom, S. E. 2004, AJ, 127, 1029

Rebull, L. M., Stauffer, J. R., Megeath, S. T., Hora, J. L., \& Hartmann, L. 2006, ApJ, 646, 297

Sills, A., Pinsonneault, M. H., \& Terndrup, D. M. 2000, ApJ, 534, 335

Stassun, K. G., Mathieu, R. D., Mazeh, T., \& Vrba, F. J. 1999, AJ, 117, 2941

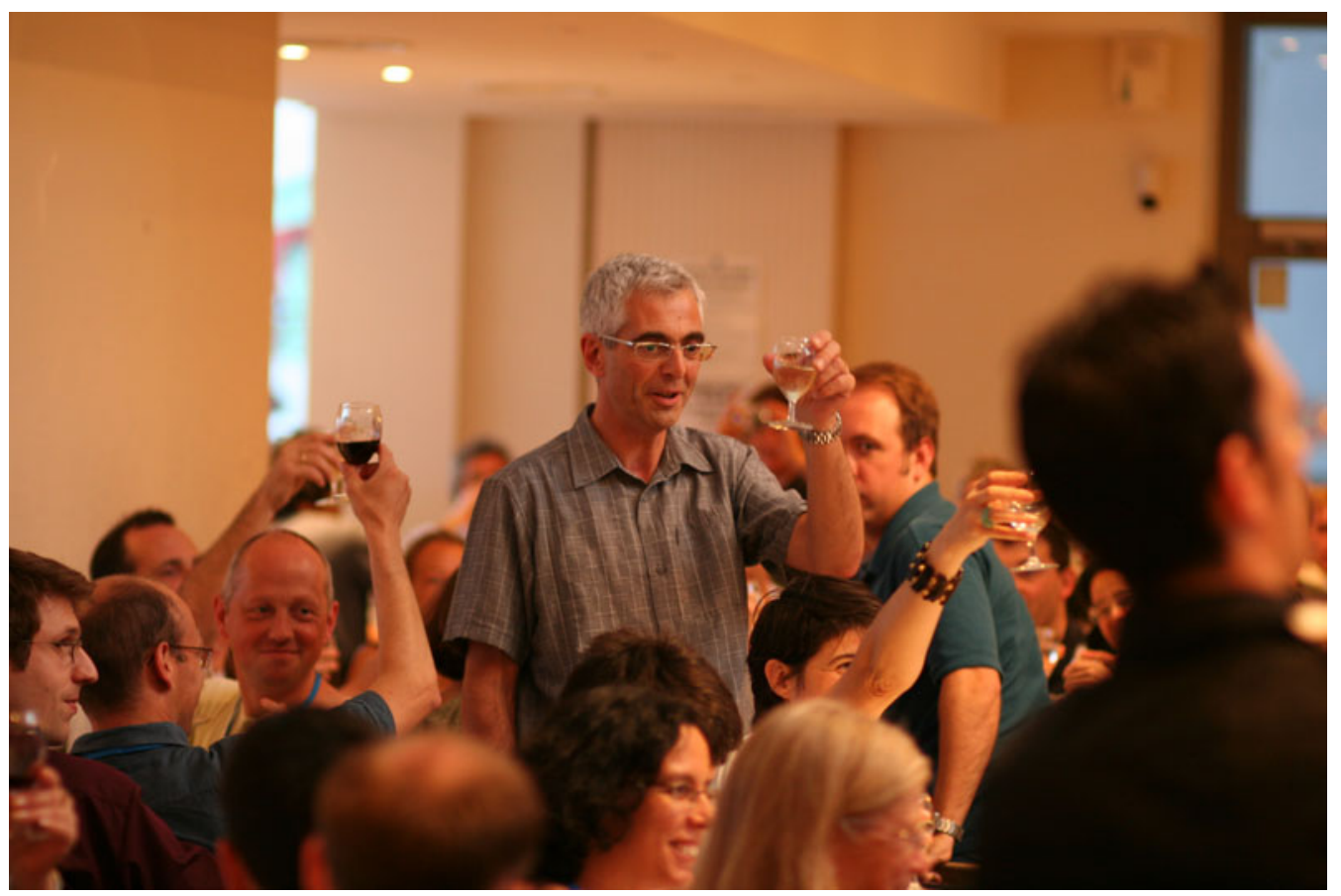

